\title{
Joint power allocation for OFDM system with cooperation at the transmitters
}

\author{
Hui Wang ${ }^{*}$, Yanzhao Hou and Yingni Zhang
}

\begin{abstract}
It is known that traditional water-filling (WF) provides a closed form solution for capacity maximization in orthogonal frequency division multiplex (OFDM) system. The solution is derived from a maximum mutual information argument with a single transmitter. Motivated by the novel technology of cooperative communication, we consider a new power allocation problem for OFDM systems with two cooperative transmitters, where each transmitter has an individual power constraint and can obtain their own perfect channel state information (CSI). The transmitters first cooperate by sharing the CSI, and then jointly optimize power allocation in the metric of sum throughput, which can be modeled as a non-convex constrained optimization problem. Through an application of Karush-Kuhn-Tucker conditions, the problem is reformulated as a convex one. Then, the closed form solution is derived with the nature of traditional WF as well as cooperative properties. Based on the derived solution, an iteration algorithm for joint water level is given for the first time, which can be explained as a cooperative WF relative to the traditional WF. Motivated by the deriving process, we extend parts of the conclusion to Ntransmitter case. Numerical results are presented to evaluate the optimal power allocation scheme in OFDM cellular system. For comparison, we also evaluate the traditional non-cooperative WF and equal power allocation scheme.
\end{abstract}

Keywords: power allocation, cooperative communication, OFDM, water-filling (WF).

\section{Introduction}

Transmit power allocation combined with rate adaptation is considered as a powerful method to increase the throughput of wireless networks [1,2]. In an orthogonal frequency division multiplex (OFDM) system, multiple receivers access a single transmitter through orthogonal subcarriers. Under a transmit power constraint at the transmitter, the traditional water-filling (WF) power allocation scheme has been proved to be optimal in the sense of maximizing the sum throughput [3]. The WF solution is derived for a maximum mutual information problem, which is widely used in OFDM system or any other scenarios that can be modeled as that multiple receivers access single transmitter through orthogonal channel [4-6], [21]. The traditional WF solutions are very simple to evaluate since all of them have a single water level and a power constraint. As a consequence, it

\footnotetext{
*Correspondence: whuissss@gmail.com

Wireless Technology Innovation Institute, Key Laboratory of University

Wireless Communication, Ministry of Education, Beijing university of Post and
} Telecommunications, Beijing, China

\section{Springer

(C) 2012 Wang et al; licensee Springer. This is an Open Access article distributed under the terms of the Creative Commons Attribution License (http://creativecommons.org/licenses/by/2.0), which permits unrestricted use, distribution, and reproduction in any medium, provided the original work is properly cited. is quite straightforward to compute them numerically in practice. In order to find the exact value of the water level, iterative WF algorithm has been proposed in many literature to compute the solutions numerically $[7,8]$.

Recently, the novel technology of cooperative communications has widely been proposed for wireless networks such as cellular networks and wireless ad hoc networks [9-12]. The essential of cooperative communications lies in that by exchanging information some individual independent transmission links or systems can merge into an equal larger link or system. Then, through jointly designing the transmit/receive structure or optimizing the recourse allocation from a global rather than local perspective, various gain can be obtained over the non-cooperative case. Moreover, in practical system, cooperative beam/resource, control, cooperative transmission, relaying, and cooperative MIMO are drawing attention as a solution to achieve high user throughput at the cell edge (and system throughput) in cellular systems. As discussed in 
standardizing groups of IMT-advanced, these technologies are expected to be essential in the next generation cellular networks.

Motivated by the concept of cooperative communication, this article considers a cooperative power allocation scheme for OFDM systems with individual independent power constraint at each transmitter. The transmitters with their own perfect channel state information (CSI) available first cooperate by exchanging the CSI, and then jointly optimize the power allocation in the metric of sum throughput (capacity). We first focus on 2-transmitter case, and then extend parts of the derived conclusion to general $\mathrm{N}$-transmitter case.

The main contribution of this article is that we obtain the closed form solution for throughput maximization for 2-transmitter case by solving a non-convex constrained optimization problem. The solution turns out to take the form of traditional WF and also combined with some regular cooperative feature. Based on the derived solution, an optimal joint WF (Jo-WF) algorithm is proposed to get the joint Jo-WF level subsequently for the first time. Motivated by the theoretical derivation of the 2-transmitter case, we also extend parts of the conclusion to arbitrary $N$-transmitter case. Numerical simulation results verify that the proposed Jo-WF power allocation provides a significant sum throughput gain over the traditional non-cooperative WF and equal power allocation (EPA). It is also concluded that when there is no cooperation between the transmitters, traditional WF is just local optimal, and the EPA is near optimal when the transmission power is high enough. Parts of this study appear in a pattern work [13].

Although the study is analyzed for OFDM system, it is emphasized that the derived solution can be also applied into any other scenarios that can be modeled as that multiple receivers access multiple transmitters through orthogonal channel in the time, space, or code domain. Considering the flexibility of transmitter's category, e.g., base station or relay station, it is known that the derived Jo-WF power allocation scheme can be valid for any cooperative networks such as next-generation cellular networks or ad hoc networks.

\section{System model}

We consider an OFDM system with $N$ cooperative transmitters and $K$ independent receivers as depicted in Figure 1 when $N=2$. We assume that all the transmitters can obtain their own perfect CSI. We also assume that all the transmitters can exchange the CSI reliably through cooperative channel between the transmitters in real time. The transmitters jointly send separate information to $K$ receivers through $K$ orthogonal subcarriers of the same spectral bandwidth as $\Delta B$. Each transmitter has an individual independent power constraint.

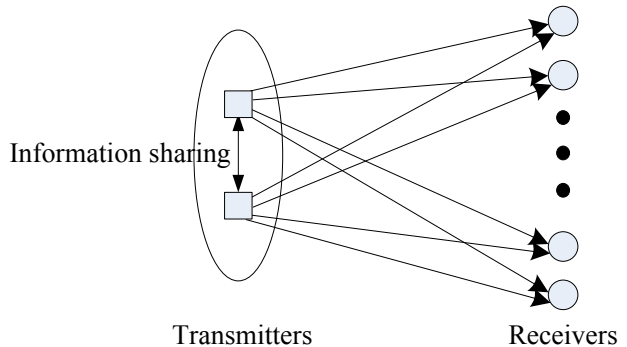

Figure 1 Two transmitters cooperative power allocation system model.

In order to focus solely on power allocation, we do not explicitly consider subcarrier scheduling here. However, it is noted that the power allocation results presented in this article are valid for any scheduling strategy, as the effect of one such strategy over another is simply to induce different subcarrier statistics for the selected subcarrier [14].

Since it is multi-carrier system, the sum of all the subcarrier's capacity is the system capacity. Therefore, supposing a certain $K$ subcarriers has been selected by arbitrary scheduling algorithm. We assume that each subcarrier is narrow enough to experience flat fading and the channel gain is constant within a given time slot. Then, the achievable throughput given by the additive white Gaussian noise (AWGN) Shannon Capacity (sum throughput) can be expressed as

$$
\begin{aligned}
& R=\sum_{k=1}^{K} \log _{2}\left(1+\frac{\sum_{n=1}^{N} P_{n k}\left|h_{n k}\right|^{2}}{N_{0} \Delta B}\right) \\
& \text { s.t. } \sum_{k=1}^{K} P_{n k}=P_{n}, n=1,2, \ldots, N, \quad P_{n k} \geq 0, \forall n, k,
\end{aligned}
$$

where $N_{0}$ represents the power spectral density of AWGN. $P_{n k}$ is the transmit power allocated from the $n$th transmitter to the $k$ th subcarrier, $P_{n}$ is the power constraint at the $n$th transmitter, and $h_{n k}$ is the corresponding subcarrier gain between the $n$th transmitter and the $k$ th subcarrier.

Consider the problem that how to allocate the power among the $K$ orthogonal narrow-band subcarriers to maximize the system throughput $R$, i.e., we search for the optimal cooperative power allocation scheme by approaching the following optimization problem

$$
\mathbf{P}_{n}^{*}=\left(P_{n, 1}^{*}, \ldots, P_{n, K}^{*}\right)=\arg \max _{\mathbf{P}_{n} \in \Omega^{K}} R, \quad n=1,2, \ldots, N
$$

where $\Omega^{K}=\left\{\mathbf{P}_{n} \mid \forall k \in(1, \cdots K), 0 \leq P_{n k} \leq P_{n}, \quad \sum_{k=1}^{K} P_{n k}=P_{n}\right\}$ is the feasible set. Since $\Omega^{K}$ is a closed and bounded set and $R: \Omega^{K} \rightarrow \Re$ is continuous, Equation (1) has a solution [[15], Theorem 0.3]. 


\section{Optimal transmit power allocation}

For notational simplicity, let $\gamma_{n k}=\left(P_{n}\left|h_{n k}\right|^{2}\right) / N_{0} \Delta B$ and $x_{n k}=P_{n k} / P_{n}$. Then, Equation (1) can be rewritten as

$$
R=\sum_{k=1}^{K} \log _{2}\left(1+\sum_{n=1}^{N} x_{n k} \gamma_{n k}\right)
$$

Thus, the throughput maximization problem can be defined as

$$
\begin{aligned}
& \max R=\sum_{k=1}^{K} \log _{2}\left(1+\sum_{n=1}^{N} x_{n k} \gamma_{n k}\right) \\
& \text { s.t. } \sum_{k=1}^{K} x_{n k}=1 \text { and } x_{n k} \geq 0, \forall n, k
\end{aligned}
$$

Note 1: Before we move on, it is emphasized that the problem (3) is a non-convex optimization problem, which can be reformulated as a convex one when $N=2$ through the application of Karush-Kuhn-Tucker (KKT) conditions [16]. In order to solve the problem from the mathematical point of view, in the following part, we first analyze a 2-transmitter case and achieve the closed form solution. Then, based on the derived solution an optimal cooperative power allocation algorithm is presented subsequently. Finally, motivated by the regular theoretical derivation of the 2-transmitter case, we extend the parts of the conclusion to arbitrary $N$-transmitter case.

\subsection{2-Transmitter case}

In this section, we analyze the 2-transmitter case. We first reformulate the problem as a convex optimization one, and then achieve the closed form solution for maximizing the sum throughput. The problem (3) can be rewritten as

$$
\begin{aligned}
& \max R=\sum_{k=1}^{K} \log _{2}\left(1+\sum_{n=1}^{2} x_{n k} \gamma_{n k}\right) \\
& \text { s.t. } \sum_{k=1}^{K} x_{n k}=1 \text { and } x_{n k} \geq 0, \forall n, k
\end{aligned}
$$

Theorem 1: The non-convex optimization problem (4) can be reformulated as the follow

$$
\begin{aligned}
& \max R=\log \left(1+\sum_{n=1}^{2} x_{n m} \gamma_{n m}\right)+\sum_{k=1, k \neq m}^{K} \log _{2}\left(1+x_{n_{k} k} \gamma_{n_{k} k}\right) \\
& \text { s.t. } m \in\{1,2, \ldots, K\}, n_{k} \in\{1,2\}, \sum_{k} x_{n k}=1 \text { and } x_{n k} \geq 0, \forall n, k
\end{aligned}
$$

which means that in order to maximize the sum throughput, only some $m$ th receiver is transmitted jointly by the two transmitters, and each other receiver is only transmitted by some single transmitter. The first term on the right-hand side represents the throughput of the receiver transmitted jointly by the two transmitters. The second term represents the sum throughput of the receivers transmitted by single transmitter. Or from mathematics perspective, the optimization problem (4) must be achieved on a specific bound domain.

Proof: The KKT conditions of the non-convex problem (4) can be expressed as

$$
\begin{array}{ll}
x_{n k} \geq 0, & \forall n \in\{1,2\}, k \in\{1,2, \ldots, K\} \\
\sum_{k=1}^{K} x_{n k}-1=0, & \forall n \in\{1,2\} \\
\lambda_{n k} \leq 0, & \forall n \in\{1,2\}, k \in\{1,2, \ldots, K\} \\
\lambda_{n k} x_{n k}=0, & \forall n \in\{1,2\}, k \in\{1,2, \ldots, K\} \\
\frac{\gamma_{n k}}{1+\sum_{l=1}^{2} x_{l k} \gamma_{l k}}-\lambda_{n k}=v_{n}, & \forall n \in\{1,2\}, k \in\{1,2, \ldots, K\}
\end{array}
$$

where $\lambda_{n k}$ and $v_{n}$ are Lagrange multiplier [16] associated with inequality constraint and equality constraint, respectively. In the following, we will prove that the optimization problem (4) can only be achieved on a specific bound domain by contradiction.

Suppose the problem (4) can be achieved in the interior of the $\operatorname{dom} R$ (domain of function $R$ ), i.e., $x_{n k}>0$, $\forall n \in\{1,2\}, k \in\{1,2, \ldots, K\}$. Then from the fourth condition in (6), it can be derived $\lambda_{n k}=0$ for any $n \in\{1$, $2\}, k \in\{1,2, \ldots, K\}$, which combined with the last condition in (6), we can get

$$
\left\{\begin{array}{l}
\frac{\gamma_{1 k}}{1+\sum_{l=1}^{2} x_{l k} \gamma_{l k}}=v_{1} \\
\frac{\gamma_{2 k}}{1+\sum_{l=1}^{2} x_{l k} \gamma_{l k}}=v_{2}
\end{array}\right.
$$

It can be further derived from (7)

$$
\frac{\gamma_{11}}{\gamma_{21}}=\frac{\gamma_{12}}{\gamma_{22}}=\cdots=\frac{\gamma_{1 K}}{\gamma_{2 K}}
$$

However, recalling the definition

$$
\gamma_{n k}=\left(P_{n}\left|h_{n k}\right|^{2}\right) / N_{0} \Delta B,
$$

substitute it into (8), we have

$$
\frac{\left\|h_{11}\right\|^{2}}{\left\|h_{21}\right\|^{2}}=\frac{\left\|h_{12}\right\|^{2}}{\left\|h_{22}\right\|^{2}}=\cdots=\frac{\left\|h_{1 K}\right\|^{2}}{\left\|h_{2 K}\right\|^{2}}
$$

Since $h_{n k}$ is constant for a given time slot, the probability of the equation above established is almost zero in practical system. Therefore, the supposition that the problem (4) can be achieved in the interior of the dom $R$ cannot hold, i.e., the optimal power allocation scheme must be achieved on the bound domain. To find the 
solution on the bound domain, without loss of generality, first we assume the problem can be solved with $x_{n_{j} k_{j}}=0$ for some $k_{j} \in\{1,2, \ldots, K\}, n_{j} \in\{1,2\}$. For notational simplicity, let

$$
\Omega=\{(n, k) \mid n \in\{1,2\}, k \in\{1,2, \ldots, K\}\} /\left\{\left(n_{j}, k_{j}\right)\right\},
$$

Then, the optimization problem (4) can be rewritten as

$$
\begin{aligned}
& \max R=\sum_{k=1, k \neq k_{j}}^{K} \log \left(1+\sum_{n=1}^{2} x_{n k} \gamma_{n k}\right)+\log \left(1+\sum_{n=1, n \neq n_{j}}^{2} x_{n k} \gamma_{n k_{j}}\right) \\
& \text { s.t. } \sum_{k=1}^{K} x_{n k}=1 \forall n \text { and } x_{n k} \geq 0 \forall(n, k) \in \Omega \text { and } x_{n, k_{j}}=0
\end{aligned}
$$

which means that there are $K-1$ receivers transmitted jointly by the two transmitters, and the $k_{j}$ th receiver is transmitted by single transmitter. The KKT conditions of the optimization problem (10) can be written as

$$
\begin{array}{ll}
x_{n k} \geq 0, & \forall\{(n, k)\} \in \Omega \\
\sum_{k=1}^{K} x_{n k}-1=0, & \forall n=1,2 \text { and } x_{n_{j} k_{j}}=0 \\
\lambda_{n k} \leq 0, & \forall\{(n, k)\} \in \Omega \\
\lambda_{n k} x_{n k}=0, & \forall\{(n, k)\} \in \Omega \\
\frac{\gamma_{n k}}{1+\sum_{l=1}^{2} x_{l k} \gamma_{l k}}-\lambda_{n k}=v_{n,} & \forall\{(n, k)\} \in \Omega \\
\frac{\gamma_{n k_{j}}}{1+\sum_{l=1, l \neq n_{j}}^{2} x_{l k_{j}} \gamma_{l k_{j}}}-\lambda_{n k_{j}}=v_{n,}, & \forall n \neq n_{j}
\end{array}
$$

Similarly, using proof by contradiction, suppose the problem (10) can be solved with $x_{n k}>0$ for any $\{(n, k)\}$ $\in \Omega$. Then from the fourth condition in (11), it can be derived $\lambda_{n k}=0$ for any $\{(n, k)\} \in \Omega$, which combined with the last condition in (11), we can get

$$
\left\{\begin{array}{l}
\frac{\gamma_{n k}}{1+\sum_{l=1}^{2} x_{l k} \gamma_{l k}}=v_{n}, \forall\{(n, k)\} \in \Omega \\
\frac{G_{n k_{j}}}{1+\sum_{l=1, l \neq n_{j}}^{2} x_{l k_{j}} \gamma_{l k_{j}}}=v_{n}, \forall n \neq n_{j}
\end{array} .\right.
$$

It can be further derived from (12)

$$
\frac{\gamma_{11}}{\gamma_{21}}=\frac{\gamma_{12}}{\gamma_{22}}=\cdots=\frac{\gamma_{1\left(k_{j}-1\right)}}{\gamma_{2\left(k_{j}-1\right)}}=\frac{\gamma_{1\left(k_{j}+1\right)}}{\gamma_{2\left(k_{j}+1\right)}}=\frac{\gamma_{1\left(k_{j}+2\right)}}{\gamma_{2\left(k_{j}+2\right)}} \cdots=\frac{\gamma_{1 K}}{\gamma_{2 K}}(13)
$$

Substitute $\gamma_{n k}=\left(P_{n}\left|h_{n k}\right|^{2}\right) / N_{0} \Delta B$ into (13), we have

$$
\begin{aligned}
\frac{\left\|h_{11}\right\|^{2}}{\left\|h_{21}\right\|^{2}}=\frac{\left\|h_{12}\right\|^{2}}{\left\|h_{22}\right\|^{2}}=\cdots=\frac{\left\|h_{1\left(k_{j}-1\right)}\right\|^{2}}{\left\|h_{2\left(k_{j}-1\right)}\right\|^{2}} \\
=\frac{\left\|h_{1\left(k_{j}+1\right)}\right\|^{2}}{\left\|h_{2\left(k_{j}+1\right)}\right\|^{2}}=\frac{\left\|h_{1\left(k_{j}+2\right)}\right\|^{2}}{\left\|h_{2\left(k_{j}+2\right)}\right\|^{2}} \cdots=\frac{\left\|h_{1 K}\right\|^{2}}{\left\|h_{2 K}\right\|^{2}}
\end{aligned}
$$

Similarly, the probability of Equation (14) established is also almost zero for a given time slot. So, the supposition that the problem (10) can be achieved with $x_{n_{j} k_{j}}=0$ and $x_{n k}>0$ for any $\{(n, k)\} \in \Omega$ cannot hold. From (8) and (13), it is indicated that if only there are $L>1$ receivers which are transmitted jointly by the two transmitters and the each other receiver is transmitted only by some single transmitter, i.e., the problem (4) can be achieved with $x_{n l}>0, \forall l \in\{1,2, \ldots, L\}, n \in\{1,2\}$, we would have

$$
\frac{\gamma_{1 k_{1}}}{\gamma_{2 k_{1}}}=\frac{\gamma_{1 k_{2}}}{\gamma_{2 k_{2}}}=\cdots=\frac{\gamma_{1 k_{L}}}{\gamma_{2 k_{L}}} .
$$

Substituting $\gamma_{n k}=\left(P_{n}\left|h_{n k}\right|^{2}\right) / N_{0} \Delta B$ into it, we can further get

$$
\frac{\left\|h_{1 k_{1}}\right\|^{2}}{\left\|h_{2 k_{1}}\right\|^{2}}=\frac{\left\|h_{1 k_{2}}\right\|^{2}}{\left\|h_{2 k_{2}}\right\|^{2}}=\cdots=\frac{\left\|h_{1 k_{L}}\right\|^{2}}{\left\|h_{2 k_{L}}\right\|^{2}}
$$

which is almost impossible in practical system. Therefore, in order to maximize the sum throughput, $L \leq 1$, that is, at most one receiver can be transmitted jointly by the two transmitters, and each other receiver is transmitted only by some single transmitter, i.e., the optimization problem (4) can be reformulated as (5).

To further verify the conclusion that in order to maximize the sum throughput, only some $m$ th receiver is transmitted jointly by the two transmitters, and each other receiver is only transmitted by some single transmitter, we present a specific example here by numerical simulation (see Figure 2). Assume a 2-receiver case, considering the path loss, shadowing and noise, for a randomly channel realization as

$$
\begin{aligned}
& \gamma_{11}=0.1901 \gamma_{12}=0.6364 \\
& \gamma_{21}=0.1365 \gamma_{22}=0.6949
\end{aligned} .
$$

Performing an exhausted search algorithm for the power allocation, the sum throughput can be obtained as Figure 2. The power constrain at the two transmitters is set the same as $30 \mathrm{dBm}$. It is observed that the maximum sum throughput is achieved at point

$$
\begin{aligned}
& x_{11}=1.0 \quad x_{12}=0.0 \\
& x_{21}=0.3 \quad x_{22}=0.7^{\prime}
\end{aligned}
$$

which is consistent with the theorem 1, i.e., only the first receiver is transmitted jointly by the two transmitters.

Theorem 2: The solution to the problem (5) takes the simple form of traditional power WF [17] results and is also characterized by cooperation.

Proof: We first prove that the optimization problem (5) is a convex optimization problem, and then achieve 


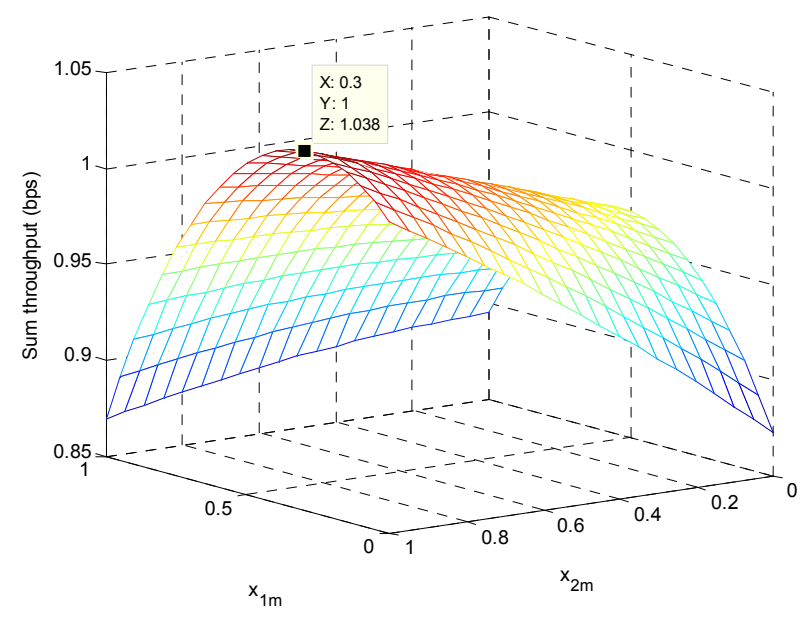

Figure 2 Sum throughput for a randomly channel realization and $P_{1}=P_{2}=30 \mathrm{dBm}$.

the solution through the application of $K K T$ conditions.

Obviously, the $\operatorname{dom} R$ in (5) is a polyhedral [16] which is a convex set. The Hesse matrix of function $-R$ is

$$
\begin{aligned}
& \nabla^{2}(-R)=\left[\begin{array}{ll}
\mathbf{A} & \mathbf{0} \\
\mathbf{0} & \mathbf{B}
\end{array}\right] \\
& \text { where } \mathbf{A}=\left[\begin{array}{c}
\frac{\gamma_{1 m}^{2}}{1+x_{1 m} \gamma_{1 m}+x_{2 m} \gamma_{2 m}} \frac{\gamma_{1 m} \gamma_{2 m}}{1+x_{1 m} \gamma_{1 m}+x_{2 m} \gamma_{2 m}} \\
\frac{\gamma_{1 m} \gamma_{2 m}}{1+x_{1 m} \gamma_{1 m}+x_{2 m} \gamma_{2 m}} \frac{\gamma_{2 m}^{2}}{1+x_{1 m} \gamma_{1 m}+x_{2 m} \gamma_{2 m}}
\end{array}\right] \\
& \mathbf{B}=\operatorname{diag}\left[\frac{\gamma_{n_{1} m}^{2}}{1+x_{n_{1} m} \gamma_{n_{1} m}} \frac{\gamma_{n_{2} m}^{2}}{1+x_{n_{2} m} \gamma_{n_{2} m}} \ldots \frac{\gamma_{n_{m-1} m}^{2}}{1+x_{n_{m-1} m} m \gamma_{n_{m-1} m}} \frac{\gamma_{n_{m+1} m}^{2}}{1+x_{n_{m+1} m} m \gamma_{n_{m+1} m}}\right. \\
& \left.\frac{\gamma_{n_{m+2} m}^{2}}{1+x_{n_{m+2} m} \gamma_{n_{m+2} m}} \cdots \frac{\gamma_{n_{K} m}^{2}}{1+x_{n_{K} m} \gamma_{n_{K} m}}\right]
\end{aligned}
$$

Obviously, arbitrary order principal minor of $\nabla^{2}(-R)$ is non-negative for any $x_{n k} \in \mathbf{d o m}-R$, i.e., $\nabla^{2}(-R)$ is positive semi-definite or $\nabla^{2}(R)$ is negative semi-definite. So, $R$ is concave [15]. To this point, we finish the proof that the problem (5) is a convex optimization problem. For analytic simplicity, we rewritten the optimization problem (5) as

$$
\begin{gathered}
\max R=\log \left(1+\sum_{n=1}^{2} x_{n m} \gamma_{n m}\right)+ \\
\sum_{k_{1}=1}^{K_{1}} \log \left(1+x_{1 k_{1}} \gamma_{1 k_{1}}\right)+\sum_{k_{2}=1}^{K_{2}} \log \left(1+x_{2 k_{2}} \gamma_{2 k_{2}}\right) \\
\text { s.t. } \sum_{k_{n}=1}^{K_{n}} x_{n k_{n}}+x_{n m}=1, x_{n m} \geq 0, x_{n k_{n}} \geq 0, \forall n, K_{1}+K_{2}+1=K
\end{gathered}
$$

where the index $k_{1}$ and $k_{2}$ represent the receivers transmitted only by transmitter ${ }_{1}$ and transmitter ${ }_{2}$, respectively, the index $m$ still represents the receiver transmitted jointly by the two transmitters. Thus, the first term in (15) represents throughput of the receiver transmitted jointly by the two transmitters, the second, and the third term represent the sum throughput of the receivers transmitted only by transmitter ${ }_{1}$ and transmit$\mathrm{ter}_{2}$, respectively. Then, the $K K T$ conditions of (15) can be expressed as

$$
\begin{array}{ll}
x_{n m} \geq 0, x_{n k_{n}} \geq 0, & \forall n \in\{1,2\} \\
\sum_{k_{n}=1}^{K_{n}} x_{n k_{n}}+x_{n m}=1, & \forall n \in\{1,2\} \\
\lambda_{n m} \leq 0, \lambda_{n k_{n}} \leq 0, & \forall n \in\{1,2\} \\
\lambda_{n m} x_{n m}=0, \lambda_{n k_{n}} x_{n k_{n}}=0, & \forall n \in\{1,2\} \\
\frac{\gamma_{n m}}{1+x_{1 m} \gamma_{1 m}+x_{2 m} \gamma_{2 m}}-\lambda_{n m}=v_{n}, & \forall n \in\{1,2\} \\
\frac{\gamma_{n k_{n}}}{1+x_{n k_{n}} \gamma_{n k_{n}}}-\lambda_{n k_{n}}=v_{n}, & \forall n \in\{1,2\}
\end{array}
$$

By directly solving these equations, the closed form solution for the optimal cooperative power allocation for the 2-transmitter case can be obtained, since it is a convex optimization problem. We start by noting that $\lambda_{n k}$ acts as a slack variable in the last equation in (16), so it can be eliminated, leaving

$$
\begin{array}{lr}
x_{n m} \geq 0, & \forall n \in\{1,2\} \\
x_{n k_{n}} \geq 0, & \forall n \in\{1,2\} \\
\sum_{k_{n}=1}^{K_{n}} x_{n k_{n}}+x_{n m}=1, & \forall n \in\{1,2\} \\
v_{n} \geq \frac{\gamma_{n m}}{1+x_{1 m} \gamma_{1 m}+x_{2 m} \gamma_{2 m}}, & \forall n \in\{1,2\} \\
v_{n} \geq \frac{\gamma_{n k_{n}}}{1+x_{n k_{n}} \gamma_{n k_{n}}}, & \forall n \in\{1,2\} \\
x_{n m}\left(\frac{\gamma_{n m}}{1+x_{1 m} \gamma_{1 m}+x_{2 m} \gamma_{2 m}}-v_{n}\right)=0, & \forall n \in\{1,2\} \\
x_{n k_{n}}\left(\frac{\gamma_{n k_{n}}}{1+x_{n k_{n}} \gamma_{n k_{n}}}-v_{n}\right)=0, & \forall n \in\{1,2\}
\end{array}
$$


If $v_{n}<\gamma_{n k_{n}}$, the fifth condition in (17) can only hold if $x_{n k_{n}}>0$, which by the last condition implies that $v_{n}=\gamma_{n k_{n}} /\left(1+x_{n k_{n}} \gamma_{n k_{n}}\right)$. Solving for $x_{n k_{n}}$, we conclude that $x_{n k_{n}}=1 / v_{n}-1 / \gamma_{n k_{n}}$. If $v_{n} \geq \gamma_{n k_{n}}$, then $x_{n k_{n}}>0$ is impossible, because it would imply $v_{n} \geq \gamma_{n k_{n}}>\gamma_{n k_{n}} /\left(1+x_{n k_{n}} \gamma_{n k_{n}}\right)$, which violates the last condition. Therefore, $x_{n k_{n}}=0$ if $v_{n} \geq \gamma_{n k_{n}}$. Thus, we have

$$
x_{n k_{n}}=\left\{\begin{array}{ll}
\frac{1}{v_{n}}-\frac{1}{\gamma_{n k_{n}}}, & v_{n}<\gamma_{n k_{n}} \\
0 & , v_{n} \geq \gamma_{n k_{n}}
\end{array}, \forall \mathrm{n} \in\{1,2\},\right.
$$

Note 2: To this point, the solution for $x_{n k_{n}}$ is achieved, and according to the third condition in (17), we can also obtain the solution for $x_{n m}$. From (18), it is observed that the power allocation for the receivers transmitted by single transmitter take the form of traditional WF results. The receivers which have better channel state will get more power allocation and the receivers which have the channel state worse than the water level should not be transmitted. To further get the value of $x_{n k_{n}}$, the water-level $v_{1}$ and $v_{2}$ must be obtained. In the following part, we proceed to get the water-level $v_{1}$ and $v_{2}$ with an iteration algorithm which is proposed for the first time.

For notational simplicity, Equation (18) can be simplified as $x_{n k_{n}}=\max \left\{0,1 / v_{n}-1 / \gamma_{n k_{n}}\right\}$, which combined with the third condition in (17) we conclude that

$$
\left\{\begin{array}{l}
\sum_{k_{1}=1}^{K_{1}} \max \left\{0,1 / v_{1}-1 / \gamma_{1 k_{1}}\right\}=1-x_{1 m} \\
\sum_{k_{2}=1}^{K_{2}} \max \left\{0,1 / v_{2}-1 / \gamma_{2 k_{2}}\right\}=1-x_{2 m}
\end{array} .\right.
$$

For the common receiver transmitted by the two transmitters, similarly, if $v_{n}<\gamma_{n m}$, the fourth condition in (17) can only hold if $x_{1 m} \gamma_{1 m}+x_{2 m} \gamma_{2 m}>0$. If $v_{n} \geq \gamma_{n m}$, then $x_{n m}>0$ is impossible, because it would imply $v_{n} \geq$ $\gamma_{n m}>\gamma_{n m} /\left(1+x_{1 m} \gamma_{1 m}+x_{2 m} \gamma_{2 m}\right)$, which violates the sixth condition. Therefore, $x_{n m}=0$ if $v_{n} \geq \gamma_{n m}$. Thus, we have

$$
\begin{cases}x_{1 m} \gamma_{1 m}+x_{2 m} \gamma_{2 m}>0, & v_{1}<\gamma_{1 m} \text { or } v_{2}<\gamma_{2 m} \\ x_{n m}=0, & v_{n} \geq \gamma_{n m} \forall n \in\{1,2\}\end{cases}
$$

To obtain the value of the water-level, first we suppose that both of $x_{1 m}$ and $x_{2 m}$ are positive, which can only hold if $v_{1}<\gamma_{1 m}$ and $v_{2}<\gamma_{2 m}$. Then, by the sixth condition in (17) we can get

$$
\begin{aligned}
& \left\{\begin{array}{l}
\frac{\gamma_{1 m}}{1+x_{1 m} \gamma_{1 m}+x_{2 m} \gamma_{2 m}}-v_{1}=0 \\
\frac{\gamma_{2 m}}{1+x_{1 m} \gamma_{1 m}+x_{2 m} \gamma_{2 m}}-v_{2}=0
\end{array},\right. \text { or } \\
& \left\{\begin{array}{l}
x_{1 m} \gamma_{1 m}+x_{2 m} \gamma_{2 m}=\frac{\gamma_{1 m}}{v_{1}}-1 \\
\frac{\gamma_{1 m}}{v_{1}}=\frac{\gamma_{2 m}}{v_{2}}
\end{array}\right.
\end{aligned}
$$

Combining (19) and (21), we can obtain

$$
\begin{array}{r}
\frac{\gamma_{1 m}}{v_{1}}+\sum_{k_{1}=1}^{K_{1}} \max \left\{0, \frac{\gamma_{1 m}}{v_{1}}-\frac{\gamma_{1 m}}{\gamma_{1 k_{1}}}\right\}+\sum_{k_{2}=1}^{K_{2}} \max \left\{0, \frac{\gamma_{1 m}}{v_{1}}-\frac{\gamma_{2 m}}{\gamma_{2 k_{2}}}\right\} \\
=\gamma_{1 m}+\gamma_{2 m}+1
\end{array}
$$

Or, put more simply

$$
\frac{\gamma_{1 m}}{v_{1}}+\sum_{n=1}^{2} \sum_{k_{n}=1}^{K_{n}} \max \left\{0, \frac{\gamma_{1 m}}{v_{1}}-\frac{\gamma_{n m}}{\gamma_{n k_{n}}}\right\}=\gamma_{1 m}+\gamma_{2 m}+1
$$

The left-hand side is a piecewise-linear decreasing function of $v_{1}$, with breakpoints at $\gamma_{1 k_{1}}$ and $\left(\gamma_{1 m} / \gamma_{2 m}\right) \gamma_{2 k_{2}}$, so the equation has a unique solution which is readily determined. There are $K_{1}+K_{2}$ breakpoints for the piecewise-linear decreasing function in the left-hand side of Equation (22), $\left\{\begin{array}{lllll}\gamma_{11} & \gamma_{12} & \ldots & \gamma_{1 K_{1}}\end{array}\right\}$ and $\left(\gamma_{1 m} / \gamma_{2 m}\right)\left\{\begin{array}{llll}\gamma_{21} & \gamma_{22} & \ldots & \gamma_{2 K_{2}}\end{array}\right\}$. For constructing the algorithm, we sort these breakpoints with ascending order as $\left\{\begin{array}{llllll}\gamma_{1} & \gamma_{2} & \ldots & \gamma_{K_{1}+K_{2}}\end{array}\right\}$. For notational simplicity, let

$$
\delta=1+\gamma_{2 m} / \gamma_{1 m}+1 / \gamma_{1 m}
$$

then Equation (22) can be rewritten as

$$
\frac{1}{v_{1}}+\sum_{k=1}^{K_{1}+K_{2}} \max \left\{0, \frac{1}{v_{1}}-\frac{1}{\gamma_{k}}\right\}=\delta
$$

which can be solved with the iteration algorithm listed in Algorithm 1. Then through second equation in (21), we can also get the solution of $v_{2}$. To this point, we achieve the solution under the assumption that both of $x_{1 m}$ and $x_{2 m}$ are positive. The assumption can only hold when $v_{1}<y_{1 m}$ and $v_{2}<y_{2 m}$, so we have to check whether the solution satisfies the assumption. If satisfying, we proceed to obtain the optimal power allocation through (18). Otherwise at least one of $x_{1 m}$ and $x_{2 m}$ should vanish, and from (20), if $v_{1} \geq y_{1 m}$ and $v_{2}<y_{2 m}$, it indicates $x_{1 m}=0$ and $x_{2 m}>0$; if $v_{1}<y_{1 m}$ and $v_{2} \geq y_{2 m}$, it indicates $x_{2 m}=0$ and $x_{1 m}>0$; if $v_{1} \geq y_{1 m}$ and $v_{2} \geq y_{2 m}$, it indicates both of $x_{1 m}$ and $x_{2 m}$ vanish. In the above three cases, since there is no receiver transmitted jointly by the two transmitters, the problem (4) can be reformulated as 


$$
\begin{aligned}
& \max C=\sum_{k_{1}=1}^{K_{1}} \log \left(1+x_{1 k_{1}} \gamma_{1 k_{1}}\right)+\sum_{k_{2}=1}^{K_{2}} \log \left(1+x_{2 k_{2}} \gamma_{2 k_{2}}\right) \\
& \text { s.t. } \sum_{k_{n}=1}^{K_{n}} x_{n k_{n}}=1, x_{n k_{n}} \geq 0 \forall n, K_{1}+K_{2}=K
\end{aligned}
$$

The first and the second terms in the right-hand side in (24) represent the sum throughput of the receivers transmitted by transmitter ${ }_{1}$ and by transmitter ${ }_{2}$, respectively. Obviously, the two terms are independent of each other, both of which can be maximized, respectively, with the traditional WF algorithm. The solutions take the form [17]

$$
x_{n k_{n}}=\max \left\{0,1 / \lambda_{n}-1 / \gamma_{n k_{n}}\right\}, \forall \mathrm{n} \in\{1,2\}
$$

Sort $\left\{\gamma_{n 1} \gamma_{n 2} \ldots \gamma_{n K_{n}}\right\}$ with ascending order as $\left\{\begin{array}{llll}\gamma_{1} & \gamma_{2} & \ldots & \gamma_{K_{n}}\end{array}\right\}$, then $\lambda_{n}$ can be obtained through solving Equation (26) with the traditional WF algorithm listed in Algorithm 2.

$$
\sum_{k_{n}=1}^{K_{n}}\left\{\max \left\{0,1 / \lambda_{n}-1 / \gamma_{k_{n}}\right\}=1, \forall n \in\{1,2\} .\right.
$$

In conclusion, the convex optimization problem (15) can be solved by Algorithm 3. To this point, the corresponding optimal cooperative algorithm is presented.

Note 3: To this point, we propose the iteration algorithm to get the value of the water level $v_{1}$ and $v_{2}$. It is noted that, if $v_{1}<\gamma_{1 m}$ and $v_{2}<\gamma_{2 m}$, i.e., there is one receiver transmitted jointly by the two transmitters, we have (21) which reflects the cooperative feature. To further analysis, the power allocation results $x_{1 n}$ and $x_{2 n}$ are not independent and they restrict each other through the water level $v_{1}$ and $v_{2}$. In addition, from (21) and the algorithm solving for water level, the two water levels $v_{1}$ and $v_{2}$ can be unified as a single global water level and the power allocation scheme can be explained as a cooperative global WF process relative to the traditional WF power allocation. Moreover, it should be emphasized that, to perform the proposed joint power allocation scheme, each transmitter must obtain all the CSI to further get all the $\gamma_{k}$, which can be realized only by cooperation between the transmitters, i.e., exchanging CSI.

\section{2. $\mathrm{N}$-transmitter case}

In this section, based on the above analysis and theorem, we extend parts of the derived conclusion to arbitrary $N$-transmitter case through mathematical derivation. Some similar assumption and analysis method are omitted here.

Theorem 3: Let $\varphi_{k}$ denote the set of the transmitters for the $k$ th receiver, $k=1,2, \ldots, K$, length $\left(\varphi_{k}\right)$ denotes the size of $\varphi_{k}$. In order to maximize the sum throughput,

$$
\text { length }\left(\phi_{k_{1}} \cap \phi_{k_{2}}\right) \leq 1, \forall k_{1} \neq k_{2} \text { and } k_{1}, k_{2} \in\{1,2, \ldots, K\}
$$

which means that there is no more than one receiver transmitted jointly by more than one transmitter.

Proof: The problem for $N$-transmitter case has been defined as (3), The $K K T$ conditions of which can be written as

$$
\begin{array}{ll}
x_{n k} \geq 0, & \forall n \in\{1,2, \ldots, N\}, k \in\{1,2, \ldots, K\} \\
\sum_{k=1}^{K} x_{n k}-1=0, & \forall n \in\{1,2, \ldots, N\} \\
\lambda_{n k} \leq 0, & \forall n \in\{1,2, \ldots, N\}, k \in\{1,2, \ldots, K\} \\
\lambda_{n k} x_{n k}=0, & \forall n \in\{1,2, \ldots, N\}, k \in\{1,2, \ldots, K\} \\
\frac{\gamma_{n k}}{1+\sum_{i=1}^{N} x_{i k} \gamma_{i k}}-\lambda_{n k}=v_{n}, \forall n \in\{1,2, \ldots, N\}, k \in\{1,2, \ldots, K\}
\end{array}
$$

Suppose that there are $L>1$ receivers transmitted jointly by $T>1$ transmitters, i.e., the problem (3) can be solved with $x_{l t}>0$ for $\forall l \in\{1,2, \ldots, L\}, t \in\{1,2, \ldots, T\}$, then from fourth condition (27), it can be derived $\lambda_{l t}=$ 0 for any $l \in\{1,2, \ldots, L\}, t \in\{1,2, \ldots, T\}$, which combined with the last equation in (27), we can get

$$
\frac{\gamma_{l t}}{1+\sum_{i=1}^{N} x_{i k} \gamma_{i k}}=v_{t}, \forall t \in\{1,2, \ldots, T\}, l \in\{1,2, \ldots, L\}
$$

It can be further derived from (28)

$$
\frac{\gamma_{t_{1} 1}}{\gamma_{t_{2} 1}}=\frac{\gamma_{t_{1} 2}}{\gamma_{t_{2} 2}}=\cdots=\frac{\gamma_{t_{1} L}}{\gamma_{t_{2} L}}, \forall t_{1}, t_{2} \in\{1,2, \ldots, T\}
$$

Substitute $\gamma_{n k}=\left(P_{n}\left|h_{n k}\right|^{2}\right) / N_{0} \Delta B$ into (29), we have

$$
\frac{\left\|h_{t_{1} 1}\right\|^{2}}{\left\|h_{t_{2} 1}\right\|^{2}}=\frac{\left\|h_{t_{1} 2}\right\|^{2}}{\left\|h_{t_{2} 2}\right\|^{2}}=\cdots=\frac{\left\|h_{t_{1} L}\right\|^{2}}{\left\|h_{t_{2} L}\right\|^{2}}
$$

which is impossible in practical system. So, the supposition cannot hold, i.e., the theorem 3 is proved.

\section{Numerical simulations}

In this section, the performance of the proposed optimal cooperative power allocation schemes is evaluated in OFDM system. The system model is depicted in Figure 3. Considering a practical application of cooperative communication to improve the cell edge throughput, we assume that the user located within the colored zone and assume that the channels between all the transmitters and receivers experience independent fading.

$R$ is $1000 \mathrm{~m}$. Non-co $R$ is $600 \mathrm{~m}$. Path loss model adopts Okumura-Hata [18]: $l(d)=137.74+35.22 \lg (d)$ in $\mathrm{dB}$, Shadowing's standard deviation is $3.65 \mathrm{~dB}$. For the sake of simplification, we assume $\Delta B=15 \mathrm{kHz}$ and the downlink noise $\mathrm{N}_{0} \Delta B$ at each subcarrier is assumed 


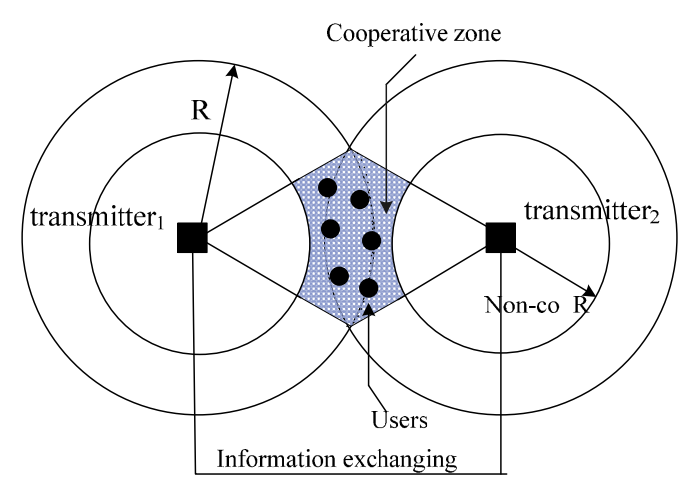

Figure 3 Simulation model, the user is located in the colored zone

to be the same as $-105 \mathrm{dBm}$. Assume that the two transmitters have the same power constraint as $P$. The numerical results are generated by averaging the throughput over 1000 randomly generated users' location realizations.

The throughput results of the optimal joint power allocation scheme are plotted in Figure 4. For comparison, we also show the results of equal power scheme (EPA) and traditional WF scheme under different number of users. It is noted that the traditional WF scheme here means each transmitter separately allocates its power by traditional solution of WF. As expected, the proposed power allocation scheme is shown to be optimal both at low-power constraint and high-power constraint, and provide a significant throughput gain. As the numbers of user increases, the gain is greater. Moreover, the crossing point in Figure 4 indicates that when the transmission power is high enough, EPA is superior to traditional WF, similar conclusion has also been pointed out in many other contributions $[19,20]$. It is concluded that when there is no cooperation between the transmitters traditional WF is just local optimal; the EPA is near optimal when the transmission power is high enough.

\section{Conclusion}

In this study, we have investigated the power allocation for OFDM system with cooperation at the transmitters. The transmitters first cooperate by exchanging the CSI, and then joint optimal power allocation. To maximize the sum throughput, at most one receiver should be jointly transmitted by the two transmitters, and each other receiver is transmitted by some single transmitter. Then, the closed form solutions to the optimal joint power allocation are achieved in the 2-transmitter case, which turn out to take the form of traditional WF and also combined with some regular cooperative feature. Based on the solution, an optimal joint power allocation algorithm is proposed subsequently for the first time, which can be explained as a joint WF relative to the traditional WF. Motivated by the derivation process in the 2-transmitter case, we extend parts of the conclusion to $N$-transmitter case. Numerical results verify the optimality of the derived scheme and show throughput gains over traditional non-coordinated WF and EPA.

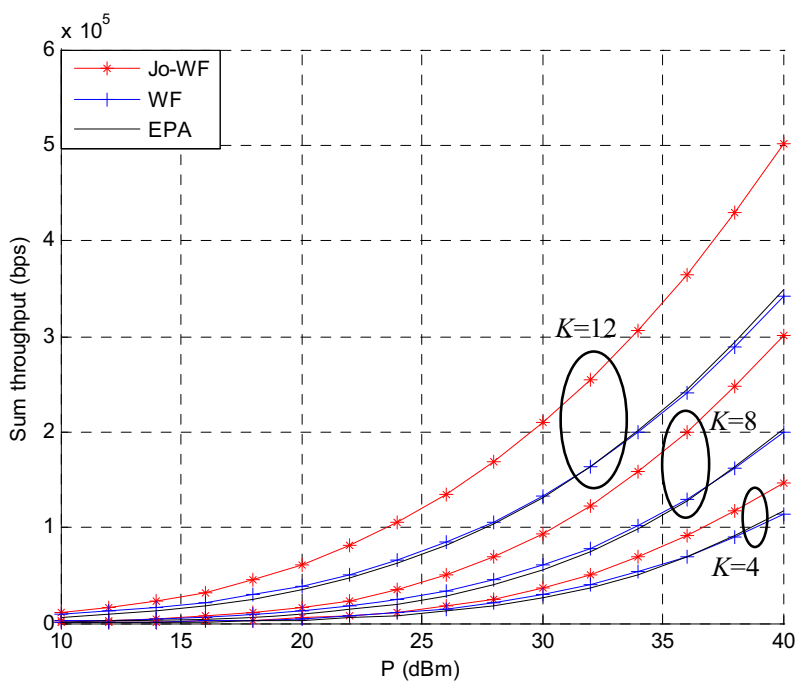

Fig. 4 2-CTP case, sum throughput versus power constraint $P$ 


\section{Algorithm 1. Jo-WF algorithm for water level}

Initialization $v_{1}=K /\left(\delta+\sum_{k=1}^{K_{1}+K_{2}}\left(1 / \gamma_{k}\right)\right)$

$i=1$

while $v_{1}>\gamma_{i}$ do

$v_{1}=(K-i) /\left(\delta+\sum_{k=i+1}^{K_{1}+K_{2}}\left(1 / \gamma_{k}\right)\right)$

$i=i+1$

\section{End}

\section{Algorithm 2. Traditional WF algorithm for water level}

Initialization $\lambda_{n}=K_{n} /\left(1+\sum_{k=1}^{K_{n}}\left(1 / \gamma_{k}\right)\right)$

$i=1$

while $v_{1}>\gamma_{i}$ do

$\lambda_{n}=\left(K_{n}-i\right) /\left(1+\sum_{k=i+1}^{K_{n}}\left(1 / \gamma_{k}\right)\right)$

$$
i=i+1
$$

\section{End}

\section{Algorithm 3. Optimal cooperative power allocation algorithm}

1. Assume both of $x_{1 m}$ and $x_{2 m}$ are positive, which can only hold if $v_{1}<\gamma_{1 m}$ and $v_{2}<\gamma_{2 m}$.

2. Solve (22) with the Jo-WF algorithm in Algorithm 1 to get $v_{1}$ and then obtain $v_{2}$ through the second equation in (21).

3. Check whether $v_{1}<\gamma_{1 m}$ and $v_{1}<\gamma_{2 m}$. If satisfying, go to step 4, otherwise, go to step 5

4. Get $x_{n k_{n}}$ through (18) and $x_{n m}$ through the third condition in (17)

5. If $v_{n} \geq \gamma_{n m}$, set $x_{n m}=0$. Then the problem can easily be solved with the traditional WF algorithm in Algorithm 2.

\section{Acknowledgements}

This study was sponsored by International Scientific and Technological Cooperation Program (2010DFA11060), National Natural Science Foundation of China (61027003), China-EU International Scientific and Technological Cooperation Program (0902)

\section{Competing interests}

The authors declare that they have no competing interests.

Received: 20 July 2011 Accepted: 30 April 2012 Published: 30 April 2012

\section{References}

1. D Tse, Fundamentals of Wireless Communication, (Cambridge University Press, Cambridge, 2005)
2. L Venturino, N Prasad, X Wang, Coordinated scheduling and power allocation in downlink multicell OFDMA networks. IEEE Trans Veh Technol. 58(6), 2835-2848 (2009)

3. AJ Goldsmith, S-G Chua, Variable-rate variable-power MQAM for fading channels. IEEE Trans Commun. 45, 1218-1230 (1997)

4. G Munz, S Pfletschinger, J Speidel, An efficient waterfilling algorithm for multiple access OFDM, in IEEE Global Telecommunications Conference, 2002, Taipei, Taiwan (GLOBECOM '02). 1, 681-685 (17-21 November 2002)

5. Y-C Liang, R Zhang, JM Cioffi, Subchannel grouping and statistical waterfilling for vector block-fading channels. IEEE Trans Commun. 54(6), 1131-1142 (2006)

6. K Kim, Efficient adaptive modulation and power allocation algorithm for OFDMA cellular systems, in IEEE WTS, Pomona, CA, United states, pp. 169-173 (April 2005)

7. G Scutari, DP Palomar, S Barbarossa, Asynchronous iterative water-filling for Gaussian frequency-selective interference channels. IEEE Trans Inf Theory. 54(7), 2868-2878 (2008)

8. G Scutari, DP Palomar, S Barbarossa, The MIMO iterative waterfilling algorithm. IEEE Trans Signal Process. 57(5), 1917-1935 (2009)

9. JN Laneman, DNC Tse, GW Wornell, Cooperative diversity in wireless networks: efficient protocols and outage behavior. IEEE Trans Inf Theory. 50(12), 3062-3080 (2004)

10. A Scaglione, DL Goeckel, JN Laneman, Cooperative communications in mobile ad hoc networks. IEEE Signal Process Mag. 23(5), 18-29 (2006)

11. Y Jing, B Hassibi, Distributed space-time coding in wireless relay networks. IEEE Trans Wirel Commun. 5, 3524-3536 (2006)

12. M-k Chang, S-y Lee, Performance analysis of cooperative communication system with hierarchical modulation over rayleigh fading channel. IEEE Trans Wirel Commun. 8(6), 2848-2852 (2009)

13. B Luo, Q Cui, H Wang, X Tao, Optimal joint water-filling for OFDM systems with multiple cooperative power sources, in IEEE Global Telecommunications Conference, Miami, FL, United states, pp. 1-5 (December 2010)

14. M Andrews, K Kumaran, K Ramanan, A Stoylar, P Whiting, R Vijayakumar, Providing quality of service over a shared wireless link. IEEE Commun Mag. 39(2), 150-154 (2001)

15. R Fletcher, Practical Methods of Optimization, Vol. 1: Unconstrained Optimization, (John Wiley \& Sons, New York, 1980)

16. S Boyd, L Vandenberghe, Convex Optimization, (Cambridge University Press, Cambridge, UK, 1985)

17. RG Gallager, Information Theory and Reliable Communication, (Wiley, New York, 1968)

18. H Holma, A Toskala, WCDMA for UMTS, (John Wiley \& Sons Ltd, New York, 2000)

19. P Viswanath, DNC Tse, V Anantharam, Asymptotically optimal water-filling in vector multiple-access channels. IEEE Trans Inf Theory. 47(1), 241-267 (2001)

20. H-W Lee, S Chong, Downlink resource allocation in multi-carrier systems: frequency-selective vs. equal power allocation. IEEE Trans Wirel Commun. 7(10), 3738-3747 (2008)

doi:10.1186/1687-1499-2012-154

Cite this article as: Wang et al:: Joint power allocation for OFDM system with cooperation at the transmitters. EURASIP Journal on Wireless

Communications and Networking 2012 2012:154.

\section{Submit your manuscript to a SpringerOpen ${ }^{\circ}$} journal and benefit from:

- Convenient online submission

- Rigorous peer review

- Immediate publication on acceptance

- Open access: articles freely available online

- High visibility within the field

- Retaining the copyright to your article

Submit your next manuscript at $\boldsymbol{s p r i n g e r o p e n . c o m ~}$ 